\title{
Discours médiés par ordinateur et stratégies d'adresse
}

\author{
Eva Havu (Université de Helsinki, Finlande)
}

\begin{abstract}
Generally, interviewers and interviewees address each other by the "traditional" deferential pronoun vous in French oral and written media, whereas in specialized magazines and television programs, $t u$ often seems to be the usual address pronoun (cf. Williams \& van Compernolle 2009; Coveney 2010). However, Internet forums discussing the use of forms of address (consulted in 2015), point out the prevalence of very traditional opinions, contrasting with the above-mentioned observation. In general, less attention has been paid to nominal forms of address.

This paper examines the use of pronominal and nominal forms of address in blogs on politics and in forums discussing current political events, that is in two types of Computer-Mediated Communication where the writers lack information about their interlocutor (age, social status, profession...). It shows to what extent their choice is dependent on the type of medium and on the context as well as on the relation between the interlocutors (conflictual, neutral...) and how nominal forms of address accentuate certain aspects of interpersonal relations. It also points out that the generalization of $t u$ in special contexts does not reflect a drastic change in the use of forms of address in general.
\end{abstract}

Keywords: French forms of address, Computer-Mediated Communication, contextual variation, blogs, discussion forums

\section{Introduction}

La recherche de Brown et Gilman (1960) sur le système d'adresse dans plusieurs langues, qui prend en compte les relations interpersonnelles entre les locuteurs (pouvoir vs. solidarité), a été un point de départ décisif pour bien des travaux sur les formes nominales et pronominales d'adresse (Monsieur, maman, les filles...; tu, vous) dans différents types d'écrit et d'oral. ${ }^{1}$ Toutefois, jusqu'à présent, les contextes d'emploi plus récents de ces formes, tels que les discours médiés par ordinateur $\left(\mathrm{DMO}^{2}\right)$, ont été moins étudiés. ${ }^{3}$

En français, deux pronoms personnels sont employés dans l'adresse directe: la forme déférente vous $(\mathrm{V})$ qui soulignait anciennement surtout une relation de pouvoir et de distance entre les locuteurs et la forme plus informelle $t u(\mathrm{~T})$ qui marque une relation de solidarité et

\footnotetext{
${ }^{1}$ Par exemple Havu 2015 (survol général) ; Suomela-Härmä, Härmä et Havu 2013 (écrit : langues romanes) ; Kerbrat-Orecchioni 2010 et 2014 (oral : français et autres langues) ; Amenós et al. 2004, Isosävi et Lappalainen 2015, Peeters et Ramiere 2009, (surtout langues parlées en Europe), etc.

${ }^{2}$ Mondada (1999) parle de communication médiatisée par ordinateur (CMO).

${ }^{3}$ Par exemple van Compernolle 2008, Williams et van Compernolle 2009 : 378 ; pour DMO, voir par exemple Barnes 2003, Helasvuo et al. 2014, Herring 2004, Lewis 2005.
} 
de familiarité ( $c f$. Brown et Gilman 1960). Même si le tutoiement s'est généralisé, entre autres, dans des communautés de travail (Lévy 2006), surtout entre gens occupant une position hiérarchique de même niveau (Havu 2013), vous est toujours considéré comme le pronom correct dans une interaction entre gens qui se connaissent peu (Halmøy 2009 ; Havu 2009, 2013). C'est également le pronom utilisé entre interviewers et interviewés dans les médias oraux et écrits d'information générale (radio, télévision, journaux), ce qui s'expliquerait par le fait que ceux qui ne sont pas présents lors de l'interaction (lecteurs, auditeurs ou spectateurs) interpréteraient un tutoiement entre les interlocuteurs comme un signe d'une relation trop familière, intime, les excluant (Claudel 2009, 173). Le rapport entre lecteurs et médias d'information serait étroitement régi par un «contrat de communication » (Charron et Jacob 1999, 7) impliquant généralement le vouvoiement. Toutefois, dans une situation spécifique, le contrat peut changer et T prendre la place de V. Lévy (2006) parle de « clans » qui ont leurs propres systèmes d'adresse marquant leur appartenance à un groupe. Les interlocuteurs peuvent faire partie de plusieurs « clans » avec des contrats différents : dans un contexte médiatique général, le contrat de communication est « cordial », et les interviewés/ lecteurs/ auditeurs ont normalement recours à des formes d'adresse traditionnelles, tandis que dans les magazines ou programmes spécialisés (« clans » de spécialistes avec un contrat de communication plus « intime »), la forme non marquée d'adresse serait bien le tutoiement (Coveney 2010 ; Williams et van Compernolle 2009). Ce principe de contrat se manifesterait dans toute interaction verbale humaine.

Le discours médiatique inclut aussi bien des interactions en présentiel que des échanges verbaux in absentia. Dans la plupart des interactions médiées par ordinateur, faute de contact direct entre les interlocuteurs et faute d'informations supplémentaires (âge, profession, statut hiérarchique, etc.), l'identification à un groupe se fonde sur d'autres critères que dans les interactions face à face, ce qui se reflète également sur les formes d'adresse utilisées. Le « contrat » est souvent non explicité et l'absence physique de l'interlocuteur ne permet pas d'observer les réactions de l'autre qui pourraient signaler un éventuel dysfonctionnement dans le discours (Codreanu et Celik 2012, 12). Parfois, cette absence d'interlocuteur direct peut mener à des libertés d'expression, même à l'utilisation de formules évitées dans une communication face à face (Tuomarla 2014, 7-8). Si, dans certains forums de discussion, les participants préfèrent maintenir les stratégies d'adresse plutôt traditionnelles qu'ils utilisent en général off-line, dans bien d'autres, un tutoiement symétrique s'est généralisé ( $c f$. Williams et van Compernolle 2009; voir aussi Isosävi 2005). Le modèle de celui qui initie la discussion semble jouer un rôle important, mais pas décisif.

Le pronom peut même changer au milieu d'un commentaire ou à l'intérieur d'une chaîne de discussion $(\mathrm{T}=>\mathrm{V} ; \mathrm{V}=>\mathrm{T})$. Même si, d'après Béal $(2009,137)$, les « allers-retours entre tutoiement et vouvoiement sont pratiquement prohibés », on les trouve dans toutes sortes de discours (par exemple Isosävi 2010, 46 ff., Kerbrat-Orecchioni 1992, 50).

Les pronoms d'adresse peuvent être accompagnés d'une forme nominale d'adresse $\left(\right.$ FNA $\left.^{4}\right)$. Ces syntagmes nominaux («apostrophes nominales », d'après Détrie 2006) « ne sont

\footnotetext{
${ }^{4}$ Kerbrat-Orecchioni (2010, 20-21) donne, entre autres, les formes nominales suivantes : noms personnels (noms de famille, prénoms, diminutifs, surnoms) ; formes d'adresse « passe-partout» (anciennement titres) : monsieur/madame/mademoiselle; titres hérités ou conférés : conte, capitaine, chef, patron ;
} 
jamais nécessaires à la complétude grammaticale de l'énonce » (Kerbrat-Orecchioni 2010, 24) et leur rôle serait donc surtout sémantique ou pragmatique. Leur valeur dépendrait, en plus de la place qu'elles occupent dans la phrase, de facteurs cotextuels (entourage linguistique, prosodique, mimo-gestuel) (Kerbrat-Orecchioni 2010, 24-25).

Les FNA assurent, d'après Kerbrat-Orecchioni (2010, 25-30), les types de fonction suivants :

I. Rôle dans l'organisation et la gestion de l'interaction :

- interpellation («valeur de hélage »);

- sélection de l'allocutaire et gestion des tours de parole.

II. Procédés de renforcement du lien interactif et de l'acte de langage, pour, entre autres, accentuer la valeur pragmatique de l'énoncé et l'obligation de réagir ( $c f$. Détrie 2006, 92) :

- rituels de politesse (salutation, remerciement) pour renforcer le caractère poli de l'énoncé ou pour adoucir une menace.

III. Rôle par rapport à la relation interpersonnelle :

- « valeurs qui résultent du sémantisme particulier d'une FNA au sein de paradigme des formes disponible » (Kerbrat-Orecchioni 2010, 28).

Ni l'éventail des FNA utilisées, ni leurs différentes valeurs pragmatiques n'ont guère été étudiées d'une manière systématique dans les discours médiés par ordinateur, très hétérogènes en soi. Pour discerner leur éventuelle variation dans les DMO, nous avons étudié deux types de discours différents, dont le premier se compose de textes rédigés par les lecteurs de la presse «traditionnelle » en ligne s'intéressant à des phénomènes politiques et le deuxième de commentaires initiés par la création d'un fil de discussion polémique publié sur un forum Internet :

1. Commentaires initiés par un blog publié dans la presse en ligne (corpus blogs) ;

2. Commentaires dans des forums de discussion traitant de différents types de questions politiques d'actualité (corpus forums).

Nous nous demanderons dans quelle mesure le choix des stratégies d'adresse dépend du type de DMO et de l'appartenance à un «clan » tutoyant ou vouvoyant, et dans quels contextes se manifestent les stratégies individuelles, non conformes à l'emploi généralisé dans la chaîne. Quant aux FNA, nous étudierons la répartition de leurs emplois pragmatiques dans les deux corpus ainsi que leur corrélation avec le pronom «majoritaire » (T ou V). Nous discuterons également les éventuelles différences entre les deux corpus.

\section{Formes d'adresse dans les corpus médiatiques examinés}

Notre étude envisage d'illustrer certaines tendances dans un échantillon de deux types de DMO (898 commentaires au total). Comme les chaînes de discussions peuvent être

noms de métier et de fonction : chauffeur, garçon (de café) ;

termes relationnels (relation de parenté, affective, professionnelle) : collègue, maman, mon fils ;

labels, qui opèrent un «catalogage » de l'interlocuteur : chers spectateurs, salut les filles;

termes affectifs, à valeur positive ou négative : chéri(e), idiot(e). 
incomplètes, par exemple parce qu'une partie des textes ont été censurés, il aurait été impossible de faire une étude strictement quantitative, prenant en compte chaque occurrence du pronom d'adresse employé. Nous avons donc seulement noté le type de pronom (T ou V) utilisé dans chaque commentaire, pour étudier de plus près le pronom d'adresse «déviant» par rapport à l'usage général dans la chaîne de discussion, ainsi que l'éventuelle alternance des pronoms. Toutes les FNA différentes ont été prises en considération.

\subsection{Chaînes de discussion initiées par un blog (corpus blogs)}

Nous avons examiné les formes d'adresse dans deux chaînes de discussion initiées par un blog ; l'un a été publié dans la version électronique du Figaro (IR) et le deuxième dans celle du Nouvel Observateur (MW) :

A. Le blog d'Ivan Rioufol: Bloc-notes : ceci n'est pas un choc de civilisations..., publié le 10 avril 2015 à 0 h01. Le blog est suivi de 606 commentaires, dont 200 ont été examinés (ci-dessous IR).

B. Le blog de Michel Wieviorka : Grèce : la politique par le bas, publié le 7 juillet 2015 à 11 h08. Le blog est suivi de 91 commentaires (ci-dessous MW).

\subsubsection{Formes pronominales d'adresse}

\subsubsection{Le pronom $T$}

Dans le deux blogs, V semble être omniprésent dans les adresses directes : parmi les 119 commentaires contenant un pronom d'adresse (sur les 291 examinés), seuls 12 (avec 6 participants différents), présentent un T. Dans 8 commentaires, $\mathrm{T}$ est adressé à un autre participant (ex. 1, 2), mais jamais à Rioufol ou Wieviorka. Dans un exemple, il s'agit (plutôt) d'une généralisation (ex. $3:$ tu $=$ on), et trois occurrences apparaissent dans une citation (exemple 4 (Bible) et 5).

\begin{tabular}{|ccccc|}
\hline Blog & T & V & $\begin{array}{c}\text { Changement } \\
\mathbf{T =}=\mathbf{V}\end{array}$ & $\begin{array}{c}\text { TOTAL des } \\
\text { commentaires }\end{array}$ \\
\hline IR & 5 & 84 & --- & 200 \\
\hline MW & 7 & 23 & 1 & 91 \\
\hline $\begin{array}{c}\text { TOTAL pr. Adresse : } \\
\text { 119 }\end{array}$ & 12 & 107 & 1 & 291 \\
\hline
\end{tabular}

Tableau I : Formes pronominales d'adresse utilisées dans les deux blogs.

\begin{tabular}{|c|c|c|c|c|}
\hline $\begin{array}{c}\text { Formes } \\
\text { pronominales } \mathbf{T}\end{array}$ & $\begin{array}{c}\text { Adresse directe à un } \\
\text { autre participant }\end{array}$ & Citation & Généralisation & $\begin{array}{c}\text { Formes } \\
\text { pronominales } \mathbf{T}\end{array}$ \\
\hline TOTAL : 12 & 8 & 3 & 1 & TOTAL : 12 \\
\hline
\end{tabular}

Tableau II : Distribution des pronoms T. 
(1) Mordraal répond à Caporal_Pancho

Bah écoute, à vue de pif et au doigt mouillé, je ne dirais pas ça, moi : [...] Tu vas me dire que je suis en voie de Amsterdammerisation et $t u$ auras raison, mais bon, plus j'y réfléchis, plus je me dis que ça peut difficilement marcher autrement. (MW)

(2) Docteur Yûl répond à Mordraal

Je serais plutôt de ton avis sur ce coup (MW)

(3) ZEN en réponse au commentaire de Jean-Louis Royer

$T u$ peux faire tout ce que tu veux, tu finis toujours par perdre, parce que c'est MOI le PATRON! (IR)

(4) ZEN en réponse au commentaire de Jeanne Espérance

Je lui demande « qui est ton Père? » (IR)

(5) PONTCARRAL

Méfiez-vous quand quelqu'un que vous détestez vous dira : «t'as d'beaux yeux, t'sais...» (IR)

\subsubsection{Changement de pronom d'adresse}

Dans un échange, $\mathrm{T}$ change en $\mathrm{V}$ : AstroZombie et MoogFest se tutoient généralement, mais quand AstroZombie critique MoogFest en parlant de « connerie », celui-ci lui répond par $\mathrm{V}$, pour signaler qu'il ne fait plus partie de son « clan »:

(6) Astro Zombie répond à MoogFest

Et ça vient couiner au hors-sujet.... Quitte à kiffer l'islamiste complotiste. Pas de doute pour ce qui est de $t a$ connerie par contre.

MoogFest répond à Astro Zombie

Êtes vous obsédé ou juste comlpètement con ? (MW)

Dans ces deux chaînes de commentaires initiées par un blog, V est donc omniprésent, T n'étant qu'occasionnellement employé par certains bloggeurs dans une adresse directe (par exemple toujours entre Mordraal et Caporal_Pancho) (blog de MW) et parfois, (blog d'IR), dans des adresses non directes (citations et généralisation).

\subsubsection{Formes nominales d'adresse (FNA)}

Dans le corpus blogs, les formes nominales d'adresse sont peu présentes en general : une seule fois, un appellatif accompagne un tutoiement explicite (citation, voir Tableau IV), les autres appellatifs se trouvant dans des contextes où les interlocuteurs se vouvoient. Seul le blog de Rioufol (IR) contient des FNA au singulier :

\footnotetext{
${ }^{5}$ Les formes pronominales ou nominales d'adresse sont en italique, tandis que les éventuels caractères gras, soulignements ou majuscules se trouvent dans l'exemple initial. Pour économiser l'espace, nous avons supprimé les heures précises de publication. Les fautes d'orthographe n'ont pas été corrigées.
} 


\begin{tabular}{cccc} 
Blog & T+FNA & V+FNA & $\begin{array}{c}\text { TOTAL des } \\
\text { commentaires }\end{array}$ \\
\hline IR & 5 & 84 & 200 \\
MW & 7 & 23 & 91 \\
\hline TOTAL FNA 39 & 12 & 107 & 291 \\
\hline
\end{tabular}

Tableau III : Formes nominales d'adresse utilisées dans les blogs.

Les FNA employés sont classables dans les trois catégories pragmatiques signalées cidessus, la catégorie II étant toutefois largement majoritaire :

\begin{tabular}{|c|c|c|}
\hline $\begin{array}{l}\text { Pronom } \\
\text { d'adresse }\end{array}$ & $\begin{array}{c}\text { Fonction } \\
\text { pragmatique de la } \\
\text { FNA }\end{array}$ & Exemples \\
\hline $\mathrm{T}$ & I : interpellation & Ami, entends-tu... (citation : Chant des partisans) \\
\hline Vsg & $\begin{array}{l}\text { I : gestion des tours de } \\
\text { parole }^{6}\end{array}$ & Mr Rioufol. Je demande que... \\
\hline Vsg & II : salutations initiales & $\begin{array}{l}\text { Bonjour mr Rioufol } \\
\text { Bonsoir anniemartine } \\
\text { Chère } m d f\end{array}$ \\
\hline Vsg & II : salutations finales & Bonne journée $m d f$ \\
\hline Vsg & II : remerciements & Merci encore Miney \\
\hline Vsg & II : excuses & Pardon madame... \\
\hline Vsg & $\begin{array}{l}\text { III : relation } \\
\text { interpersonnelle }\end{array}$ & $\begin{array}{l}\text { Mais oui, Alberto, c'est la version... } \\
\text { Très fort, Monsieur Rioufol } \\
\text { Je ne peux imaginer, Ivan Rioufol, que vous croyiez... } \\
\text { Eh oh Isabelle,... } \\
\text { L'inventaire du Front National, madame, est de voir... }\end{array}$ \\
\hline
\end{tabular}

Tableau IV : Fonctions pragmatiques des FNA utilisées ${ }^{7}$ :

I. Interpellations, sélection de l'allocutaire et gestion des tours de parole ;

II. Rituels de politesse ;

III. Relation interpersonnelle.

Dans les commentaires examinés, les formes nominales d'adresse accompagnent presque exclusivement $\mathrm{V}$ ou se trouvent dans un contexte $\mathrm{V}^{8}$. Ils accompagnent surtout des rituels de politesse.

\footnotetext{
${ }^{6}$ Les stratégies de gestion des tours de parole sont très proches des salutations initiales, étant donné que dans les deux cas l'énonciateur signale aux autres à qui est adressé le message. Nous avons inclus dans la catégorie II uniquement les énoncés contenant une marque explicite signalant qu'il s'agit d'un «rituel de politesse » (bonjour, pardon, bonne soirée...).

${ }^{7}$ Cf. Introduction, rappel du classement de Kerbrat-Orecchioni (2010, 25-30).
} 


\subsection{Forums traitant de questions politiques d'actualité (corpus forums)}

Nous avons examiné six chaînes de discussion publiées dans trois forums différents en 2015. Le nombre total des commentaires est de 607, dont 171 contiennent un pronom d'adresse ${ }^{9}$ :

\section{Chaîne de discussion}

TOTAL des commentaires

Réfugié de trois ans noyé (RTN)

Accueil des migrants (ADM)

Allemagne: une femme politique poignardée sur le marché par un anti-migrants (AFP)

Devoir sur l'islam: pour ou contre, besoin d'avis d'anciens musulmans ou connaisseurs (DIPC)

Tableau V : Distribution des commentaires dans les chaînes de discussion.

\subsubsection{Formes pronominales d'adresse}

$\mathrm{Si}$, dans les commentaires des deux blogs, V était le pronom d'adresse (quasi) omniprésent, dans les forums examinés, le tutoiement l'emporte de loin sur le vouvoiement : 154 commentaires avec T vs. 17 commentaires avec V. Dans trois échanges, le pronom initial change au milieu de l'interaction.

$\begin{array}{ccccc}\begin{array}{c}\text { Chaîne de } \\ \text { discussion }\end{array} & \text { T } & \mathbf{V} & \begin{array}{c}\text { Changement } \\ \mathbf{T}=>\mathbf{V}, \mathbf{V}=\mathbf{T}\end{array} & \begin{array}{c}\text { TOTAL des } \\ \text { commentaires }\end{array} \\ \text { RTN } & 9 & 2 & --- & 40 \\ \text { ADM } & 18 & 5 & 2 & 64 \\ \text { AFP } & 23 & --- & --- & 42 \\ \text { DIPC } & 7 & --- & --- & 13 \\ \text { MFM } & 27 & 4 & --- & 306 \\ \text { QEM } & 70 & 6 & 1 & 607 \\ \text { TOTAL pr. } & 154 & 17 & 3 & \end{array}$

Tableau VI : Formes pronominales d'adresse utilisées dans les six chaînes de discussion.

\footnotetext{
${ }^{8}$ Étant donné que les rares participants « tutoyants » ont été repérés, nous rangeons les FNA sans pronom dans le groupe des pronoms d'adresse « majoritaires » $(\mathrm{V})$.

${ }^{9}$ Nous remercions Reetta Lahtinen, étudiante en master 2 en 2015-2016, qui a recueilli le corpus forum.
} 
Dans deux chaînes de discussion (AFP, DIPC), le pronom d'adresse est régulièrement $\mathrm{T}$, tandis que dans les quatre autres apparaissent quelques adresses directes avec $\mathrm{V}$. Comme dans les blogs, nous examinerons ci-dessous seulement le choix du pronom «déviant », c'està-dire $\mathrm{V}$, ainsi que les cas de changement de pronom.

\subsubsection{Le pronom $\mathrm{V}$}

Le pronom $\mathrm{V}$ est surtout employé par certains participants et plus courant dans les critiques que dans les commentaires consensuels. Cependant, nous ne pouvons pas savoir s'il s'agit du marquage d'un désaccord (prise de distance du «clan ») ou plutôt de personnes en soi «vouvoyantes", d'autant plus que le pronom d'adresse manque souvent dans les réponses. Par exemple dans la chaîne MFM, Ajita Maitreya vouvoie je ne suis pas denis, mais comme celle/celui-ci ne s'adresse pas à elle directement, nous ne pouvons pas savoir quel serait le pronom que celle/celui-ci utiliserait :

(7) je ne suis pas denis* a écrit :

Un blabla que je résume en une phrase: les français savent que l'islam c'est de la merde.

Ajita Maitreya a écrit :

Vous feriez mieux de mettre une majuscule à "Français" et d'éviter d'extrapoler l'étron que vous êtes ...

je ne suis pas denis* a écrit :

Depuis quand « français » est un nom propre ? C'est un adjectif, bourricot.

Ajita Maitreya a écrit :

Prenez donc une grammaire élémentaire et vous verrez (avec beaucoup d'application) que dans ce cas c'est considéré comme un nom propre et prend donc une majuscule ... (MFM)

V n'est pas la marque régulière d'une prise de position critique, car la plupart des expressions de désaccord sont accompagnées de $\mathrm{T}$ :

(8) nidhalll a écit à Amysage :

Ce que tu donnes ne sont que tes propres avis sur la question et non pas des arguments irréfutables. (DIPC)

\subsubsection{Changement de pronom d'adresse}

Le changement du T au V (2 échanges), ou du V au T (1 échange) au milieu d'un échange semble être un signe de désaccord et marquer la volonté de se distancier de l'interlocuteur. Dans l'échange suivant, abyssy28 tutoie d'abord solveig121:

(9a) abyssy 28 a écrit à solveig 121

Si $t u$ travaillais pour les personnes en difficultés, mal logées voire pas logées du tout, $t u$ saurais qu'il ne suffit pas de leur ouvrir la porte de notre maison pour leur permettre de s'en sortir. Cette naiveté est déconcertante. [...] (ADM)

Dans sa nouvelle réponse à solveig121 (dont la réponse au message antérieur manque), abyssy 28 utilise V: 
(9b) Merci pour ces insultes gratuites et ces diffamations qui montrent encore une fois quel genre de citoyenne vous êtes. (ADM)

Solveig121 répond maintenant par une adresse directe, mais en se servant de T :

$(9 \mathrm{c})$

Une citoyenne

qui travaille, qui paient ses impôt, qui participe à l'effort collectif, qui a élevé ses enfants de manière à ce qu'ils deviennent eux aussi des citoyens respectables et qui respecte les biens d'autrui !

tu peux en dire autant? (ADM)

Dans sa réponse, abyssy 28 conserve V, tout en ajoutant des émoticônes :

(9d)

En effet, la majorité des gens fait ça, solveig $\Theta$

Je parlais de vie citoyenne, pas de vie de mère qui ramène des sous à sa descendance.

Vous ne comprenez même pas ce que le mot citoyenneté veut dire, on part de loin avec vous. Les impôts, on vous oblige à les payer. [...] (ADM)

Dans un autre échange de commentaires, sevim13 vouvoie d'abord son interlocuteur shirakuma en exprimant son accord, mais la/le tutoie après un long message où shirakuma la/le critique sévèrement en la/le tutoyant :

(10) sevim13 a écrit à shirakuma:

Desolé mais OUI des membres des familles royale SAOUDIENNE et QATARIS ont financer DAESH.

Ensuite oui vous avez raison les sunnite et chiite ne s'entendent pas mais rien n'empêchent ses petromonarchies a accueillir des chrétiens d'orient par exemple. [...] (QEM)

shirakuma a écit à sevim13 :

Desolé mais OUI des membres des familles royale SAOUDIENNE et QATARIS ont financer

DAESH.

Bah non. Pourquoi a ton avis ? [...]

$T u$ n'as pas l'air comprendre la géopolitique de Proche Orient. C'est pire que l'AfriqueEurope réunis, le proche orient, c'est une véritable poudrière. je te conseille d'aller voir sur wiki sur proche orient depuis démembrement de l'empire Ottoman, c'est la que ca commence [...] (QEM)

sevim13 a écrit à shirakuma :

Une nouvelle fois «des membres des familles royales » pas la famille royale.

Puisque $t u$ a l'air de connaître la region tu sais tres bien qu'a l'intérieur des familles c'est LA GUERRE pire que chez les LE PEN et certain pour déstabiliser la region ont financer

DAESH c'est un fait. [...] (QEM)

Dans les trois échanges, le changement de pronom est clairement lié à l'expression d'un désaccord, au fait de vouloir signaler que l'interlocuteur est exclu de la relation d'adresse «normale » établie antérieurement.

\subsubsection{Formes nominales d'adresse}

Les FNA accompagnent majoritairement un tutoiement (ou apparaissent dans un contexte de tutoiement, $c f$. note 7), ce qui est normal, étant donné que c'est le pronom le plus 
courant. Dans 5 commentaires seulement, le V est accompagné d'une forme nominale d'adresse.

\begin{tabular}{|cccc|}
$\begin{array}{c}\text { Chaîne de } \\
\text { discussion }\end{array}$ & T+FNA & V+FNA & $\begin{array}{c}\text { TOTAL des } \\
\text { commentaires }\end{array}$ \\
\hline RTN & 3 & 2 & 40 \\
\hline ADM & 2 & 1 & 64 \\
\hline AFP & 7 & --- & 42 \\
\hline DIPC & --- & --- & 13 \\
\hline MFM & 5 & --- & 142 \\
\hline QEM & 48 & 2 & 306 \\
\hline TOTAL FNA : 70 & 65 & 5 & 607 \\
\hline
\end{tabular}

Tableau VII : Formes nominales d'adresse dans les forums de discussion.

Quant aux fonctions pragmatiques des appellatifs, les chaînes de discussion présentent quelques différences par rapport aux blogs :

$\begin{array}{ccc}\text { Pronom } & \text { Fonction } & \\ \text { d'adresse } & \text { pragmatique de la } & \text { Exemples }\end{array}$

\begin{tabular}{|c|c|c|}
\hline Vsg & $\begin{array}{l}\text { I: gestion des tours de } \\
\text { parole }\end{array}$ & $\begin{array}{l}\text { je ne vous jette pas la pierre, Pierre }[\ldots] \text { Ills ne partent pas au vu et } \\
\text { su de tous, tranquilos Carlos. } \\
\text { @SHIRAKUMA }\end{array}$ \\
\hline Vsg & $\begin{array}{l}\text { III : relation } \\
\text { interpersonnelle }\end{array}$ & En effet, la majorité des gens fait ça, solveig \\
\hline $\mathrm{T}$ & $\begin{array}{l}\text { I: gestion des tours de } \\
\text { parole }\end{array}$ & $\begin{array}{l}\text { Ah, je viens juste de voir ton message, zazounette } \\
\text { Pour tes amis chocolat laxter } \\
\text { FOUMrack Tu fais du plagiat de pseudo maintenant } \\
\text { Donc tu va prendre combien de clandestin ou SDF chez toi } \\
\text { Mebahelle? }\end{array}$ \\
\hline $\mathrm{T}$ & II: excuses $(?=>$ III $)$ & Désolée crème mais il en faudrait beaucoup pour convaincre \\
\hline $\mathrm{T}$ & $\begin{array}{l}\text { III : relation } \\
\text { interpersonnelle }\end{array}$ & $\begin{array}{l}\text { Encore un cinglé qui a pensé comme toi, miss tare ! } \\
\text { Quand des musulmans égorgent, ce sont des désespérés, en effet } \\
\text { hein Cromagnonne? } \\
\text { Ok calme toi connard... } \\
\text { Ouh la Jean là tu te trompes de cliente }\end{array}$ \\
\hline
\end{tabular}

Tableau VIII : Fonctions pragmatiques des formes nominales d'adresse ${ }^{10}$ : (continue)

\footnotetext{
${ }^{10} \mathrm{Cf}$. note 7 .
} 
I. Interpellations, sélection de l'allocutaire et gestion des tours de parole ;

II. Rituels de politesse ;

III. Relation interpersonnelle.

La FNA accompagnant T (ou se trouvant dans un contexte $\mathrm{T}, c f$. note 7) a surtout une fonction dans l'expression d'une relation interpersonnelle, mais elle s'emploie également dans la gestion des tours de parole, comme dans les rares exemples se situant dans un contexte V. Quant aux rituels de politesse, on n'en trouve guère, car même dans l' « excuse » citée dans le tableau ci-dessus, il s'agit plutôt d'une expression d'ironie qui ferait donc partie de la catégorie III. Aucune salutation initiale/finale explicite n'apparaît dans le corpus.

\subsection{Comparaison des deux corpus}

\subsubsection{Pronoms d'adresse}

Même si les «sous-corpus » inclus dans les corpus blogs et forums ne sont pas homogènes, on peut considérer les deux corpus comme des échantillons de deux types distincts de DMO. Le choix des pronoms d'adresse y est tout à fait contraire : si, dans les commentaires des deux blogs publiés dans la presse en ligne, le pronom d'adresse est presque régulièrement $\mathrm{V}$, dans les forums de discussion, il s'agit majoritairement de $\mathrm{T}$. Le choix de la forme «minoritaire» dépend surtout de certains participants individuels, dont le nombre réduit ne permet pas de généralisations, mais une différence très nette se manifeste entre les deux corpus : dans le corpus blogs, un tiers des formes «déviantes », c'est-à-dire des T, se trouvent dans des citations ou généralisations, tandis que dans le corpus forums, la forme « déviante » $(\mathrm{V})$ représente toujours une adresse directe. Les rares cas où le pronom change au milieu d'un échange de commentaires s'expliquent dans les deux corpus par la volonté de se détacher d'un « contrat de communication » antérieur pour signaler un désaccord.

\subsubsection{Formes nominales d'adresse}

Les deux corpus se ressemblent en ce qu'ils contiennent un nombre assez réduit de FNA, mais les contextes pragmatiques où celles-ci se trouvent se distribuent d'une manière différente :

\begin{tabular}{clcc}
$\begin{array}{c}\text { Pronom } \\
\text { d'adresse }\end{array}$ & Fonction pragmatique & $\begin{array}{c}\text { Commentaires } \\
\text { blogs }\end{array}$ & $\begin{array}{c}\text { Forums de } \\
\text { discussion }\end{array}$ \\
\hline Vsg & I: gestion des tours de parole & $\mathrm{x})$ & $\mathrm{x}$ \\
\hline Vsg & II: salutations initiales & $\mathrm{x}$ & --- \\
\hline Vsg & II : salutations finales & $\mathrm{x}$ & --- \\
\hline Vsg & II : remerciements & $\mathrm{x}$ & --- \\
\hline Vsg & II: excuses & $\mathrm{x}$ & $\mathrm{x}$ \\
\hline Vsg & III : relation interpersonnelle & $\begin{array}{c}(\mathrm{x})(1 \\
\text { exemple : }\end{array}$ & --- \\
\hline
\end{tabular}




$\begin{array}{clcc} & & \text { citation) } & \\ \mathrm{T} & \text { I: gestion des tours de parole } & --- & \mathrm{x} \\ \mathrm{T} & \text { II: salutations initiales } & --- & -- \\ \mathrm{T} & \text { II: excuses } & --- & \text {--- (plutôt III) } \\ \mathrm{T} & \text { III : relation interpersonnelle } & --- & \mathrm{x}\end{array}$

Tableau IX : Fonctions pragmatiques des formes nominales d'adresse : comparaison.

Les emplois les plus courants dans le corpus blogs sont les salutations/excuses/remerciements (catégorie II) accompagnés de $\mathrm{V}$, tandis que dans le corpus forums, il s'agit surtout des catégories I et III (gestion des tours de parole et expressions de relations interpersonnelles (surtout conflictuelles)) où la FNA se trouve dans un contexte T.

La catégorie I (gestion des tours de parole) peut chevaucher avec les salutations (initiales ; catégorie II, $c f$. note 6), étant donné qu'une salutation du type bonjour $X$ peut également être interprété comme une stratégie pour signaler le destinataire ; toutefois, nous avons compté parmi les salutations uniquement les énoncés contenant une marque explicite (bonjour $X$, bonne soirée $Y$, pardon, $Z$ ). Dans le corpus forums, les FNA n'accompagnent jamais une salutation (initiale ou finale) explicite et les éventuelles autres expressions « polis » marquent plutôt l'ironie (type III).

\section{Conclusion}

Les deux corpus examinés se distinguent tout d'abord par le type de texte à la base des commentaires (blog du type article de presse vs prise de position polémique), ce qui a un impact sur le «contrat de communication » entre les membres des deux «clans » exprimant leur opinion, et donc sur le choix du pronom d'adresse. Cependant, une petite minorité des commentaires manifestent des préférences individuelles (par exemple l'utilisation de la forme standard, également choisie off line; $c f$. Halmøy 2009, 111) et, dans des situations conflictuelles, la volonté de se détacher du contrat de communication initial.

La comparaison des deux types de corpus montre qu'on ne peut pas parler d'un $\mathrm{T}$ généralisé dans les discours médiés par ordinateur, et l'emploi fréquent de $\mathrm{T}$ dans certains types de DMO ne reflète pas encore un changement radical dans les stratégies d'adresse en général. Dans les versions électroniques du Nouvel Obs, du Point et de Paris Match ${ }^{11}$, T est extrêmement rare, n'apparaissant que dans des généralisations (si tu veux faire qc, tu peux...) ou dans la citation des paroles de gens occupant une position hiérarchiquement " supérieure » et adressées à des « subordonnés »: surtout jeunes, délinquants, athlètes ou immigrés.

Dans les forums de discussion traitant de l'emploi des formes d'adresse et consultés durant l'été $2015^{12}$, les commentaires sur l'emploi des pronoms d'adresse confirment en grande partie la présence de $\mathrm{T}$ dans les forums de discussion, tout en restant parfois assez conservateurs, surtout quand il s'agit d'autres contextes (dans les offres d'emploi sur LinkedIn, T est mal perçu). Le choix du pronom d'adresse « correct » semble donc toujours être assez difficile. Il y a une dizaine d'années, Halmøy affirmait (2009 : 112-113) : «le

\footnotetext{
${ }^{11}$ Consultés en 2015 à l'aide du moteur de recherche PORC.

${ }^{12}$ Voir références dans la bibliographie.
} 
vouvoiement n'est pour l'instant pas en voie de disparition ». [...] «En France, le tutoiement généralisé ne semble toujours pas pour demain. ». Cette étude montre que ces remarques sont toujours d'actualité.

\section{Bibliographie}

Amenós, José, Christophe Campos, Dennis Davy, et Mat Pires, éds. 2004. Second-Person Pronouns and Forms of Address in the Language of Contemporary Europe. Paris: British Institute in Paris.

Barnes, Susan B. 2003. Computer-mediated communication: Human-to-Human communication across the Internet. Boston: Allyn and Bacon.

Béal, Catherine. 2009. "L'évolution des termes d'adresse en français contemporain: un essai de modélisation.»In Tu ou Vous, l'embarras du choix, édité par Bert Peeters et Nathalie Ramière, 115-45. Limoges: Editions Lambert-Lucas.

Brown, Roger, et Albert Gilman. 1960. "The pronouns of power and solidarity." In Readings in the sociology of language, édité par Joshua A. Fishman, 252-75. Hague, the Netherlands: Mouton \& Co. Printers.

Charron, Jean et Loïc Jacob. 1999. «Énonciation journalistique et subjectivité : les marques de changement.» Les études de Communication publique 14: 1-70.

Claudel, Chantal. 2009. «Tu et vous dans l'interview de presse écrite.» In $\mathbf{T u}$ ou Vous, l'embarras du choix, édité par Bert Peeters et Nathalie Ramière, 149-74. Limoges : Editions Lambert-Lucas.

Codreanu, Tatiana \& Christelle Combe Celik. 2012. «La médiation de l'interaction pédagogique sur une plateforme de visioconférence poste à poste.» ALSIC (Apprentissage des langues et systèmes d'information et de communication),Vol. 15, $\mathrm{n}^{\circ}$ 3. https://alsic.revues.org/2572

Coveney, Aidan. 2010. "Vouvoiement and tutoiement: Sociolinguistic reflections." Journal of French Language Studies, 20. 2: 127-150.

Détrie, Catherine. 2006. De la non-personne à la personne: l'apostrophe nominale. Paris : CNRS Editions.

Halmøy, Odile. 2009. «La concurrence Tu /Vous en français contemporain: paramètres, polarités, paradoxes.» In Tu ou Vous, l'embarras du choix, édité par Bert Peeters et Nathalie Ramière, 99-113. Limoges, Editions Lambert-Lucas.

Havu, Eva. 2009. «L'emploi des pronoms d'adresse dans sept villes francophones.» In $\boldsymbol{T u}$ ou Vous, l'embarras du choix, édité par Bert Peeters et Nathalie Ramière, 81-98. Limoges: Editions Lambert-Lucas.

Havu, Eva. 2013. «L'emploi des pronoms d'adresse dans un corpus français.» In Représentations des formes d'adresse dans les langues romanes, édité par Elina Suomela-Härmä, Juhani Härmä, et Eva Havu, 69-87. Helsinki: Société Néophilologique de Helsinki. 
Havu, Eva. 2015. "Forms of address." In The International Encyclopedia of Language and Social Interaction, édité par Karen Tracy, Cornelia Ilie, et Todd Sandel. New Jersey: Wiley-Blackwell.

Havu, Eva, Hanna Lappalainen, et Johanna Isosävi. 2014. «Les stratégies d'adresse en finnois: comparaison entre deux types de corpus oraux institutionnels.» In S'adresser à autrui: les formes nominales d'adresse dans une perspective comparative interculturelle, édité par Catherine Kerbrat-Orecchioni, 303-336. Chambéry: Publications Chambéry.

Helasvuo, Marja-Liisa, Marjut Johansson, et Sanna-Kaisa Tanskanen, éds. 2014. Kieli verkossa, Näkökulmia digitaaliseen vuorovaikutukseen [Language on the internet: perspectives on digital interaction]. Helsinki: Suomalaisen kirjallisuuden seura.

Herring, Susan C. 2004. "Online communication: Through the lens of discourse." In Internet Research Annual, vol. 1, édité par Mia Consalvo, Nancy Baym, Jeremy Hunsinger, Klaus Bruhn Jensen, John Logie, Monica Murero, et Leslie Regan Shade, 65-76. New York: Peter Lang.

Isosävi, Johanna. 2010. Les formes d'adresse dans un corpus de films français et leur traduction en finnois. Helsinki: Mémoires de la Société Néophilologique LXXIX.

Isosävi, Johanna. 2013. «Les valeurs sémantiques et pragmatiques des formes d'adresse françaises dans un corpus cinématographique.» In Représentations des formes d'adresse dans les langues romanes, édité par Elina Suomela-Härmä, Juhani Härmä, et Eva Havu, 109-142. Helsinki: Société Néophilologique de Helsinki.

Isosävi, Johanna. 2015. «Le dissensus, la polémicité et la violence verbale - quelles sont leurs manifestations dans la communication Facebook ? Exemple du profil "Contre Nicolas Sarkozy”.» Mémoires de la Société Néophilologique de Helsinki XCIII: 111-124.

Isosävi, Johanna et Hanna Lappalainen, éds. 2015. Saako sinutella vai täytyykö teititellä? Tutkimuksia eurooppalaisten kielten puhuttelukäytännöistä [Addressing people with T or V? Studies on Address Practices in European Languages]. Helsinki: Suomalaisen Kirjallisuuden Seura.

Kerbrat-Orecchioni, Catherine. 1992. Les interactions verbales t. II. Paris: A. Colin.

Kerbrat-Orecchioni, Catherine, éd. 2010. S'adresser à autrui. Vol. 1: Les formes nominales d'adresse en français. Chambéry: Université de Savoie.

Kerbrat-Orecchioni, Catherine, éd. 2014. S'adresser à autrui. Vol. 2: Les formes nominales d'Adresse dans une perspective interculturelle. Chambéry: Université de Savoie.

Lévy, Lionel. 2006. «On se dit tu? Si vous voulez.» Stratégies 1427: 62. www.strategies.fr/archives/1427/page_37336/management_on_se_dit_tu_si_vous_vou $\underline{\text { lez_html. }}$

Lewis, Diana M. 2005. "Arguing in English and French asynchronous online discussion." Journal of Pragmatics, 37: 1801-1818.

Mondada, Lorenza. 1999. «Formes de séquentialité dans les courriels et les forums de discussion. Une approche conversationnelle de l'interaction sur Internet.» Apprentissage des Langues et Systèmes d'Information et de Communication, 2 (1): 325. https://edutice.archives-ouvertes.fr/edutice-00000179

Peeters, Bert, et Nathalie Ramière, éds. 2009. Tu ou Vous, l'embarras du choix. Limoges: Editions Lambert-Lucas. 
Suomela-Härmä, Elina, Juhani Härmä, et Eva Havu, éds. 2013. Représentations des formes d'adresse dans les langues romanes. Helsinki: Mémoires de la Société Néophilologique de Helsinki.

Tuomarla, Ulla. 2015. «La recontextualisation et la circulation d'insultes dans les médias : le cas des commentaires des lecteurs sur des articles/vidéos publiés en ligne.»Cahiers de praxématique 63|2014, 28/12/2015. http://praxematique.revues.org/2420; DOI:10.4000/praxematique.2420.

van Compernolle, Rémi A. 2008. "Second-person pronoun use and address strategies in online personal ads from Quebec." Journal of Pragmatics 40/12: 2062-2076.

Williams, Lawrence et Rémi A. van Compernolle. 2009. "Second-person pronoun use in French language discussion fora." Journal of French Language Studies, 19: 361-378.

\section{Corpus}

\section{Corpus blogs (consulté en avril-août 2015):}

Le blog d'Ivan Rioufol: Bloc-notes: ceci n'est pas un choc de civilisations..., publié le 10 avril 2015 à 0h01 (http://blog.lefigaro.fr/rioufol/2015/04/ceci-nest-pas-un-choc.html).

Le blog de Michel Wieviorka : Grèce : la politique par le bas, publié le 7 juillet 2015 à $11 \mathrm{~h} 08$ (http://blogs.rue89.nouvelobs.com/wieviorka/2015/07/07/grece-la-politique-par-lebas-234777).

\section{Corpus forums (consulté en septembre-décembre 2015):}

Réfugié de trois ans noyé (RTN)

Forum : Aufeminin, 3/9-7/9 2015, 40 commentaires

Accueil des migrants (ADM)

Forum : Aufeminin, 5/9-8/9 2015, 64 commentaires

Allemagne : une femme politique poignardée sur le marché par un anti-migrants (AFP)

Forum : Aufeminin, 18/10-19/10 2015, 42 commentaires

Devoir sur l'islam : pour ou contre, besoin d'avis d'anciens musulmans ou connaisseurs (DIPC)

Forum : Aufeminin, 21/9-30/9 2015, 13 commentaires

Une majorité de Français hostile à l'accueil des migrants (MFM)

Forum : Doctissimo, 3/9-9/9 2015, 142 commentaires

Les quotas européens de migrants (QEM)

Forum : Jeuxvideos, 17/5-16/9, 306 commentaires

Sites consultés en juin-août 2015 (formes d'adresse):

http://communaute.wikia.com/wiki/Forum:Tutoiement_ou_vouvoiement_\%3F

http://www.developpez.net/forums/d1389247/emploi-etudes-informatique/emploi/ssii/chezalten-tutoiement-rigueur/

http://sims3.forumactif.fr/t121-debat-le-vouvoiement-ou-le-tutoiement-sur-les-forums

http://www.jeuxvideo.com/forums/1-51-48004570-1-0-1-0-le-tutoiement-et-levouvoiement.htm 
http://www.languefrancaise.net/forum/viewtopic.php?id=378\%29

http://community.norton.com/fr/forums/tutoiement

https://openclassrooms.com/forum/sujet/tutoyer-ou-vouvoyer-sur-internet-84152

http://forum.psychologies.com/psychologiescom/Ados/ados-tutoiement-sujet_1214_1.htm

Moteur de recherche PORC : http://h158.it.helsinki.fi/ 